\title{
ORIGINAL
}

\section{Polymyxin B hemoperfusion in endotoxemic septic shock patients without extreme endotoxemia: a post hoc analysis of the EUPHRATES trial}

\author{
D. J. Klein ${ }^{{ }^{*}}$, D. Foster ${ }^{2}$, P. M. Walker ${ }^{2}$, S. M. Bagshaw ${ }^{3}$, H. Mekonnen ${ }^{4}$ and M. Antonelli ${ }^{5}$
}

(c) 2018 The Author(s)

\begin{abstract}
Purpose: The EUPHRATES trial examined the impact of polymyxin B hemoperfusion (PMX) on mortality in patients with septic shock and endotoxemia, defined as $E A A \geq 0.60$. No difference was found in 28-day all-cause mortality. However, the trial showed that in some patients with septic shock the burden of endotoxin activity was extreme $(E A A \geq 0.9$ ). In a post hoc analysis, we evaluated the impact of PMX use in patients with septic shock and endotoxin activity measured between 0.6-0.89.

Methods: Post-hoc analysis of the EUPHRATES trial for the 194 patients with EAA $\geq 0.6-0.89$ who completed two treatments (PMX or sham). The primary end point was mortality at 28 days adjusted for APACHE II score and baseline mean arterial pressure (MAP). Additional end points included changes in MAP, cumulative vasopressor index (CVI), median EAA reduction, ventilator-free days (VFD), dialysis-free days (DFD) and hospital length of stay. Subpopulations analyzed were site and type of infection and those with norepinephrine dose $>0.1 \mathrm{mcg} / \mathrm{kg} / \mathrm{min}$ at baseline.

Results: At 28 days, 23 patients of 88 (26.1\%) in the PMX group died versus 39 of 106 (36.8\%) in the sham group [risk difference $10.7 \%, \mathrm{OR} 0.52,95 \% \mathrm{Cl}(0.27,0.99), P=0.047]$. When unadjusted for baseline variables, $P=0.11$. The 28 -day survival time in the PMX group was longer than for the sham group [HR $0.56(95 \% \mathrm{Cl} 0.33,0.95) P=0.03]$. PMX treatment compared with sham showed greater change in MAP [median (IQR) $8 \mathrm{mmHg}(-0.5,19.5)$ vs. $4 \mathrm{mmHg}(-4.0$, 11) $P=0.04]$ and VFD [median (IQR) 20 days $(0.5,23.5)$ vs. 6 days $(0,20), P=0.004]$. There were no significant differences in other end points. There was a significant difference in mortality in PMX-treated patients with no bacterial growth on culture [PMX, 6/30 (20\%) vs. sham, 13/31 (41.9\%), $P=0.005]$. The median EAA change in the population was $-12.9 \%$ (range: increase $49.2 \%$-reduction $86.3 \%$ ). The mortality in the above median EAA change group was PMX: 6/38 (15.7\%) vs. sham 15/49 (30.6\%), $P=0.08$.
\end{abstract}

Conclusions: These hypothesis-generating results, based on an exploratory post hoc analysis of the EUPHRATES trial, suggest measurable responses in patients with septic shock and an EAA $\geq 0.6$ to 0.89 on changes in mean arterial pressure, ventilator-free days and mortality.

Trial registration: Clinicaltrials.gov Identifier: NCT01046669. Funding Spectral Medical Incorporated.

\footnotetext{
*Correspondence: kleind@smh.ca

1 Department of Critical Care, St. Michael's Hospital, University of Toronto, 4-054c Donnelly Wing, Toronto, ON M5B1W8, Canada
}

Full author information is available at the end of the article

\section{县 Springer}


Keywords: Sepsis, Septic shock, Endotoxin, Endotoxemia, Polymyxin B, Hemoperfusion, Precision medicine

\section{Introduction}

Endotoxin is a potent and common driver of septic shock, yet results of large multi-centered trials targeting endotoxin in sepsis have been disappointing [1-6]. This is despite numerous small trials that demonstrate a survival as well as physiologic benefits in improving blood pressure (MAP) and other contributors to multiple organ failure. An important innovation in this field has been the development of the Endotoxin Activity Assay $\left(\mathrm{EAA}^{\mathrm{TM}}\right)$, which allows for rapid measurement of endotoxin activity. Elevated EAA levels have been shown to predict severe sepsis and other adverse outcomes, including death [7]. In addition, high EAA levels are found in only up to $50-70 \%$ of patients with septic shock. Therefore, failure to measure EAA may have led to missed opportunity to target patients with anti-endotoxin therapies who are more likely to benefit [7].

PMX is an extracorporeal device that selectively adsorbs endotoxin and effectively removes it from the bloodstream [8]. PMX has been used in Japan for treatment of patients with presumed gram-negative sepsis and endotoxemia for $>20$ years [9]. The standard of care is two 2-h hemoperfusion sessions approximately $24 \mathrm{~h}$ apart (Toray Industries, Tokyo, Japan). PMX therapy has been suggested to improve survival and other end points in small randomized controlled trials (RCT) such as EUPHAS [10]. Contrarily, a recent RCT in patients with postoperative intra-abdominal septic shock done in France (Abdomix trial) failed to show a mortality benefit [11]. However, both of these trials had limitations identified, indicating the need for further evidence. The EUPHRATES trial (Evaluating the Use of Polymyxin B Hemoperfusion in a Randomized controlled trial of Adults Treated for Endotoxemia and Septic Shock) aimed to enrich the target population to receive PMX by including only patients with high endotoxin activity $(E A A \geq 0.60)$ to increase the probability of benefit $[12$, 13]. In the intention-to-treat and per-protocol populations randomized in the EUPHRATES trial, no significant benefit was found in the primary end point of mortality at 28 days [13].

To better understand whether selected patients with septic shock may derive benefit from treatment with PMX, we performed a hypothesis-generating post hoc analysis of the EUPHRATES trial, focused on the heterogeneity in baseline EAA levels, using the approach recommended by Pocock and Stone [14]. Our rationale is based on recent data describing how the EAA may encounter an asymptotic "threshold effect" when endotoxin activity levels are $>0.90$ [15]. This would imply that in selected patients with EAA values $\geq 0.90$, the endotoxin activity burden may be very high with up to 100 -fold variability and is potentially unquantifiable; these patients are potentially less likely to respond to a standard regimen of two PMX treatments.

Therefore, we hypothesized in this exploratory study that in a population within the EUPHRATES trial there may be a detectable response to PMX in those patients without extreme endotoxemia at baseline.

\section{Materials and methods}

The full protocol of the EUPHRATES trial and the results have previously been published (Clinicaltrials.gov ID: NCT01046669) [13]. All patients in the EUPHRATES trial or their substitute decision-maker provided informed consent, and the trial protocol was approved by the relevant institutional research ethics board. All trial procedures were conducted in accordance with rules for Good Clinical Practice.

\section{Population}

In the EUPHRATES trial, all patients had septic shock, as defined by the receipt of vasopressors above a threshold dose of $0.05 \mathrm{mcg} / \mathrm{kg} / \mathrm{min}$ of norepinephrine equivalent for at least 2 consecutive h, plus had to be receiving antibiotics for a confirmed or presumed infection and have evidence of an additional organ dysfunction, adequate fluid resuscitation and an $\mathrm{EAA} \geq 0.60$. After the planned interim analysis, eligibility criteria were modified to include only patients with a Multiple Organ Dysfunction Score $($ MODS $)>9$. The EUPHRATES trial statistical analysis plan (SAP) defined a per-protocol population (PP) to enable analysis for randomized patients who received two PMX treatments (or sham) without major protocol violations. For this post hoc analysis, we evaluated the subgroup of PP patients with a baseline EAA in the range of $0.6-0.89$ [13].

\section{Endotoxin activity analysis}

The EAA was cleared by the US FDA in 2003; it has been approved in Canada and received the CE mark in Europe more than 10 years ago. It is the only available assay for in vivo measurement of endotoxin activity. The analytical sensitivity and specificity are reported in the package insert (Spectral Medical Inc., Toronto, Canada). The relationship between endotoxin measurement (LPS in ng/ $\mathrm{ml}$ ) and endotoxin activity (EA values, no units) is a nonlinear dose-response curve [15]. Evaluating the efficacy 
of the PMX by directly measuring the change in EAA from pre- to post-PMX treatment proved to be complex as numerous other clinical factors may impact EAA levels in septic shock. Therefore, we sought to understand whether there was a difference in treatment response in those patients who had substantial EAA reduction from pre- to post-PMX treatment. To do so, we calculated the median reduction of endotoxin in the population and compared the difference in mortality for the PMX cartridge group and the sham group for patients who were above the median reduction of endotoxin at post-treatment day 3.

\section{Mortality end point}

The primary end point was mortality at 28 days post randomization. No power calculation was done for this post hoc analysis. The data for this end point were compared between the treatment groups using chi-squared method adjusted for baseline MAP and APACHE II scores using logistic regression. Unadjusted analysis was also performed. Survival time for patients above median EAA reduction were performed using Cox proportional hazards, adjusted for baseline MAP and APACHE II. Additional analyses included comparison of mortality between the two groups at 90 days as well as KaplanMeier survival time using log-rank testing.

\section{Secondary end points}

Secondary end points included: change in organ dysfunction from baseline to day 3 measured using the MODS, change in MAP from baseline to day 3 and change in vasopressor requirements from baseline to day 3. Vasopressors were assessed using a composite scoring system, the Cumulative Vasopressor Index (CVI) [12]. Additional end points included length of stay in hospital (LOS), days alive and free of mechanical ventilation (VFD) and days alive and free from dialysis (DFD).

\section{Subgroup analyses}

Selected subgroups were assessed for mortality at 28 days. The subgroups consisted of patients according to type of infection (i.e., those with a documented gramnegative or gram-positive infection, patients with both a documented gram-negative and gram-positive infection, and patients where there was no bacterial growth on cultures). We also analyzed subgroups by site of infection (lung, intra-abdominal, bacteremia, other). There was no clinical adjudication of culture results or source control. In addition, we analyzed the subgroup with baseline norepinephrine dose above and below $0.1 \mathrm{mcg} / \mathrm{kg} / \mathrm{min}$.

\section{Safety analysis}

All adverse events (AEs) that occurred during or after the onset of treatment administration were summarized using frequency counts and percentages, by treatment group. AEs were classified by severity grade (mild, moderate, severe, life threatening, death). All serious adverse events (SAE) were assessed according to relationship to study treatment (not related, possible, definite). Study treatment was defined by three components, the PMX cartridge, dialysis catheter and intravenous heparin. A patient was counted only once.

\section{Statistical methods}

Statistical analyses were performed using $\mathrm{SAS}^{\circledR}$ (Statistical Analysis System) for Windows, version 9.3. Descriptive statistics ( $n$, mean, standard deviation, median, minimum and maximum) were calculated by treatment group for continuous variables. Frequencies and percentages were presented by treatment group for categorical variables. Survival analysis, with censoring at 28 days and 90 days, was performed using a KaplanMeier curve and the log-rank test. $P<0.05$ was considered significant, and all $P$ values were reported to two decimal places. Adjustment for multiple comparisons was not performed.

\section{Results}

\section{Population}

There were 194 patients in the per-protocol group with baseline EAA between 0.60 and 0.89 (Fig. 1). Baseline characteristics are shown in Table 1 . The most common sites of infection were intra-abdominal [68/194 (35.1\%)] and lung [67/194 (34.5\%)]. Baseline culture results from all sites showed that gram-positive infections were present for 53/194 (27.3\%) patients, and gram-negative infections were present for 36/194 (18.6\%) patients. The proportion of patients where all obtained cultures showed no growth of bacteria was 61/194 (31.4\%).

\section{Endotoxin and mortality}

There was a significant reduction in 28-day mortality (adjusted for APACHE II and baseline MAP as described) for patients with baseline EAA intervals from 0.6 to 0.89 [23/88 (26.1\%) vs. 39/106 (36.8\%) (difference - 10.7, OR $0.5295 \%$ CI $(0.27,0.99), P=0.047]$. In the unadjusted analysis the difference was not statistically significant $(P=0.11)$. Treatment with PMX versus sham in those with EAA 0.6-0.89 was associated with a significant difference in Kaplan-Meier survival time to 28 days between the groups [HR $0.56,95 \%$ CI $(0.33,0.95), P=0.03$ ]. The improvements in adjusted mortality were consistent to 90 days (HR 0.57, 95\% CI 0.35, 0.93), $P=0.02$ ) (Fig. 2). 


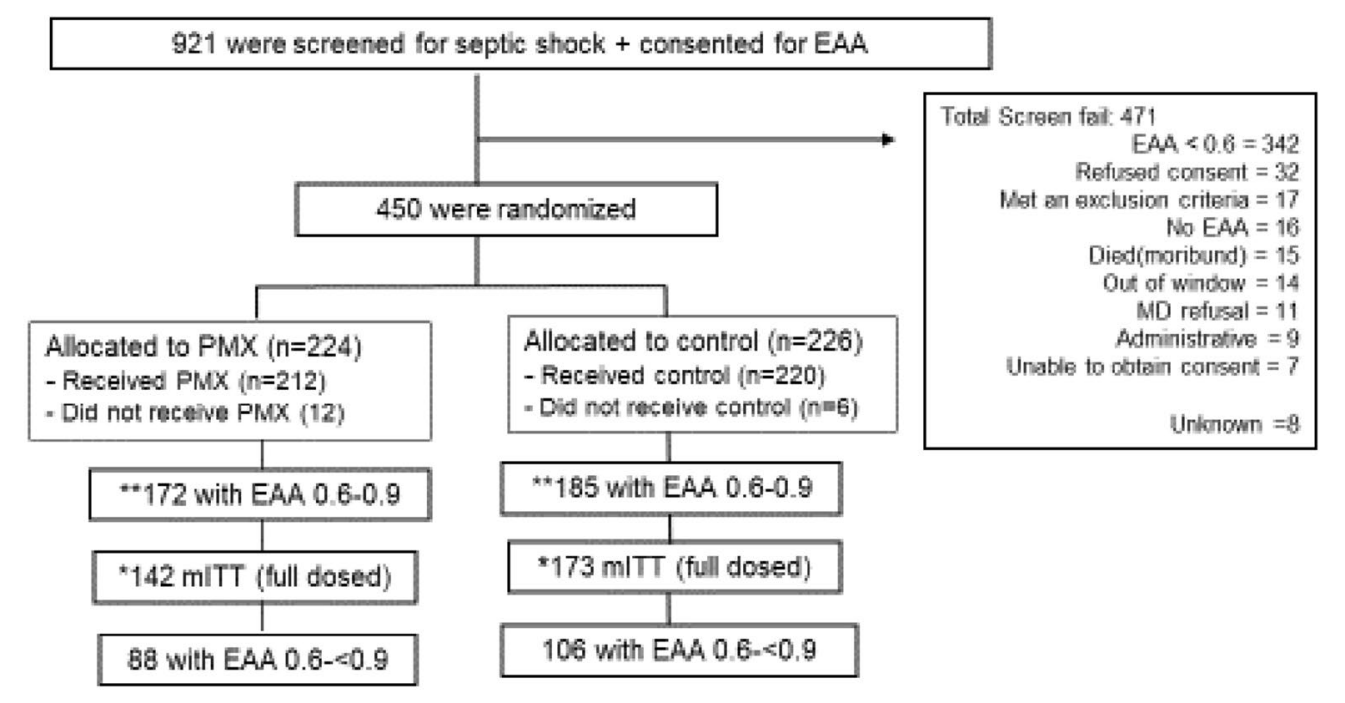

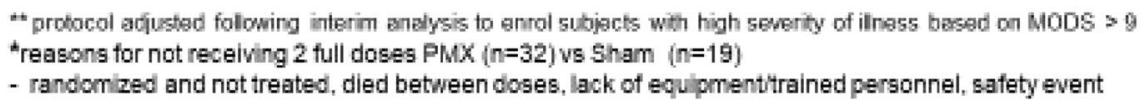

Fig. 1 Consort diagram

The median EAA change in the population was $-12.9 \%$ (range: increase $49.2 \%$-reduction $86.3 \%$ ). The adjusted hazard ratio for death in the above median EAA change group compared with the below median EAA change group was 0.38 (95\% CI, 0.15 to 0.98 ; $P=0.04$ ). Mortality at 28 days in the above median reduction group was PMX 6/38 (15.8\%) vs. sham 15/49 (30.6\%), $P=0.08$. There was no difference in EAA levels from day 1 to day 3 between PMX and sham (Supplementary Table 1 ).

\section{Secondary end points}

Treatment with PMX was associated with a significant change in median (IQR) MAP $[8(-0.5,19.5)$ vs. $4(-4.0$, 11) $P<0.05]$ and median (IQR) ventilator-free days to 28 days $[20(0.5,23.5)$ vs. $6(0,20), P=0.004]$. Changes in CVI were not statistically different, PMX -4.2 (3.5) vs. sham -3.5 (4.0), $P=0.19$. The comparison of the PMX and sham treatment groups showed no statistical difference in the median number of days alive and free of dialysis ( 20 days vs. 11 days; $P=0.59$ ), change of MODS [ -3.1 (3.7) vs. $-2.4(3.5), P=0.18]$ or length of hospital stay [PMX, 22.0 days vs. sham 28 days; $P=0.15$ ].

\section{Subgroup analyses}

Patients with baseline norepinephrine $>0.1 \mathrm{mcg} / \mathrm{kg} / \mathrm{min}$ had a statistically significant 28 -day mortality reduction with PMX treatment compared with sham [PMX: 17/69 $(24.6 \%)$ vs. sham $34 / 85(40.0 \%), P=0.02]$. Mortality at
28 days based on source of infection was: intra-abdominal source [PMX $3 / 25$ (12\%) vs. sham 11/43 (26\%) $P=0.18$ ] and lung source [PMX 10/31 (32\%) vs. sham $16 / 38$ (42\%), $P=0.40]$. For patients where cultures from any site reported no bacterial growth, there was a statistically significant benefit in 28-day mortality for the PMX group 6/30 (20\%) vs. sham 13/31 (41.9\%), $P=0.04]$.

\section{Safety results}

In the PMX treatment group, $72.7 \%$ of patients (64/88) had reported adverse events, while in the sham group, $61.3 \%$ of patients $(65 / 106)$ had reported adverse events. The most common adverse event for both groups was worsening of sepsis/septic shock; in the PMX group there were 13 such events in 88 patients (15\%) and sham, 16 events in 106 patients (16\%). The incidence of AEs is presented by system organ class (SOC) in Supplementary Table 1 . There was one serious adverse event (SAE) considered by the study site investigator to be related to use of the PMX cartridge (hypotension), and none were reported for the sham group. Two SAEs were considered to be related to the dialysis catheter in the PMX treatment group (DVT and venous embolism) and none in the sham group. Three SAEs were related to heparin use reported for the PMX group [anemia, embolism and heparin-induced thrombocytopenia) and one SAE reported for the sham treatment group (subarachnoid hemorrhage). The incidence of SAEs is presented by system organ class (SOC) in Supplementary Table 2. 


\begin{tabular}{|c|c|c|}
\hline & $\operatorname{PMX}(n=88)$ & Sham $(n=106)$ \\
\hline $\mathrm{Age}^{\mathrm{a}}[$ mean, (SD)] & $58.7(15.0)$ & $57.5(14.4)$ \\
\hline \multicolumn{3}{|l|}{ Gender $n(\%)$} \\
\hline Male & $55(62.5)$ & $66(62.3)$ \\
\hline Female & $33(37.5)$ & $40(37.7)$ \\
\hline \multicolumn{3}{|l|}{ Race $n(\%)$} \\
\hline Caucasian & $72(81.8)$ & $81(76.4)$ \\
\hline Black & $8(9.1)$ & $8(7.6)$ \\
\hline Hispanic & $3(3.4)$ & $7(6.6)$ \\
\hline Asian & $2(2.3)$ & $6(5.7)$ \\
\hline Other & $3(3.4)$ & $4(3.8)$ \\
\hline Mean arterial pressure [mean, (SD)] & $71.7(9.2)$ & $73.5(10.1)$ \\
\hline APACHE [mean, (SD)] & $30.6(7.6)$ & $29.2(8.1)$ \\
\hline $\begin{array}{l}\text { Bilirubin (mg/dl) } \\
\text { [mean, (SD)] }\end{array}$ & $0.91(0.91)$ & $1.14(1.53)$ \\
\hline Platelet count $(\times 10 \wedge 3 / \mu \mathrm{l})$ [mean, (SD)] & $155(125)$ & $130(83)$ \\
\hline $\begin{array}{l}\text { Creatinine }(\mathrm{mg} / \mathrm{dl}) \\
\text { [mean, (SD)] }\end{array}$ & $2.55(1.56)$ & $2.71(1.3)$ \\
\hline P02/Fi02 ratio [mean, (SD)] & $206(127)$ & $211(127)$ \\
\hline$n,(\%)$ ventilated & $86(97.7)$ & $105(99.1)$ \\
\hline $\begin{array}{l}\text { Heart rate (bpm) } \\
\text { [mean, (SD)] }\end{array}$ & $98(18)$ & $100(19)$ \\
\hline $\begin{array}{l}\text { Hemoglobin }(\mathrm{g} / \mathrm{dl}) \\
\text { [mean, (SD)] }\end{array}$ & $10.6(2.2)$ & $10.6(2.3)$ \\
\hline $\begin{array}{l}\text { White blood cells }(\times 10 \wedge 3 / \mu l) \text { [mean, } \\
(\text { SD)] }\end{array}$ & $17.0(11.7)$ & $17.0(13.5)$ \\
\hline \multicolumn{3}{|l|}{ Microorganisms, $n(\%)$} \\
\hline No growth & $28(31.8)$ & $31(29.3)$ \\
\hline Gram negative & $22(25.0)$ & $13(12.3)$ \\
\hline Gram positive & $20(22.7)$ & $33(31.1)$ \\
\hline Other & $5(5.7)$ & $7(6.6)$ \\
\hline Mixed & $13(14.8)$ & $22(20.8)$ \\
\hline \multicolumn{3}{|l|}{ Bacteremia $n(\%)$} \\
\hline Yes & $26(29.9)$ & $33(31.4)$ \\
\hline No & $61(70.1)$ & $72(68.6)$ \\
\hline \multicolumn{3}{|l|}{ Presumed site of infection $n(\%)$} \\
\hline Intra-abdominal & $25(29.1)$ & $43(40.6)$ \\
\hline Lung & $29(33.7)$ & $38(35.9)$ \\
\hline Mixed & $4(4.7)$ & $6(5.7)$ \\
\hline Other & $28(32.6)$ & $19(17.9)$ \\
\hline \multicolumn{3}{|l|}{ EAA categories $n(\%)$} \\
\hline 0.60 to 0.69 & $36(40.9)$ & $42(39.6)$ \\
\hline 0.70 to 0.79 & $24(27.3)$ & $37(34.9)$ \\
\hline 0.80 to 0.89 & $28(31.8)$ & $27(25.5)$ \\
\hline \multicolumn{3}{|l|}{ RRT $n(\%)$} \\
\hline Yes & $19(21.6)$ & $27(25.5)$ \\
\hline No & $69(78.4)$ & $79(74.5)$ \\
\hline \multicolumn{3}{|l|}{ Norepinephrine dosage $n$ (\%) } \\
\hline 0 to $\leq 0.05$ & $4(4.6)$ & $3(2.8)$ \\
\hline 0.05 to $\leq 0.1$ & $12(13.6)$ & $12(11.3)$ \\
\hline Above 0.1 & $69(78.4)$ & $85(80.2)$ \\
\hline
\end{tabular}

Table 1 (continued)

\begin{tabular}{|c|c|c|}
\hline & $\operatorname{PMX}(n=88)$ & Sham $(n=106)$ \\
\hline Corticosteroid use $n(\%)$ & $54(61.8)$ & $69(64.1)$ \\
\hline \multicolumn{3}{|l|}{ AKI stages $n(\%)$} \\
\hline No AKI & 19 (21.6) & $20(18.9)$ \\
\hline 1 & $9(10.2)$ & $17(16.0)$ \\
\hline 2 & $11(12.5)$ & $12(11.3)$ \\
\hline 3 & $49(55.7)$ & $57(53.8)$ \\
\hline \multicolumn{3}{|c|}{$\begin{array}{l}\text { There were no significant between-group differences in the characteristics at } \\
\text { baseline for any comparison }\end{array}$} \\
\hline \multicolumn{3}{|c|}{$\begin{array}{l}\text { APACHE Acute Physiology, Age and Chronic Health Evaluation II, EAA endotoxin } \\
\text { activity assay, RRT renal replacement therapy, AKI acute kidney injury }\end{array}$} \\
\hline
\end{tabular}

\section{Discussion}

This post hoc analysis of the EUPHRATES trial attempts to address the disparity in the existing literature regarding the potential efficacy of PMX cartridge use in septic shock. In this study, we suggest that when strict patient selection criteria are applied, including high severity of illness (MOD score $>9$ ) and an endotoxin activity level as measured by EAA between 0.6 and 0.89, PMX use is associated with an absolute mortality benefit over sham patients of $10.7 \%$ at 28 days. This finding is supported by benefit across selected subgroups and secondary end points such as for MAP, VFD and survival to 90 days. Another key aspect of our study rests in improving blood pressure accompanied by a trend to decreased use of vasopressors to reduce the burden of shock on organ dysfunction. The use of high doses of catecholamines in patients with septic shock has been associated with higher mortality and complications [16].

The approach taken in this study was to focus on the subgroup with a targeted baseline endotoxin burden range of EAA 0.6 to 0.89 . This was based on the discovery that emerged after the start of EUPHRATES that both the assay readout and associated endotoxin activity burden and the dosing of only two columns were potentially inadequate for patients with extreme endotoxemia. To our knowledge this is the first report to specifically address the impact of the burden of endotoxin activity in septic shock patients and association with outcomes. For some patients the burden is beyond the ability to accurately measure with the EAA at $>0.90$ (approximately $>4000 \mathrm{pg} / \mathrm{ml}$ of a standard endotoxin preparation of E. coli strain O111:B4) [15]. Similar threshold effects are found in many other assays including troponin, creatine kinase and TSH [17]. Therefore, unlike other antiendotoxin sepsis studies where endotoxin levels were not measured, in EUPHRATES the step forward was the recognition that in some patients an extreme burden of 
endotoxin might be present, confounding the association between PMX treatment and outcome.

The understanding of the complexity of endotoxin biology in humans continues to evolve. The concept that a specific biomarker profile in a subpopulation is critical for treatment effectiveness or lack thereof is well established in other disciplines. In oncology, this has been part of the development of epidermal growth factor receptor (EGFR), anaplastic lymphoma kinase (ALK) and programmed cell death protein-ligand 1 (PD-L1) inhibitors for lung cancer patients with specific genetic tumor mutations [18]. These mutations were not well understood when the drugs were first tested [19]. Determining the correct regimen for an anti-endotoxin strategy in septic shock may thus require a tailored, patient-focused approach.

It was unexpected that for patients with baseline EAA levels $\geq 0.90$ the burden of endotoxin activity was so high that conventional prescription of PMX (2 treatments in $24 \mathrm{~h}$ ) did not provide benefit. Future studies to address the population with extreme endotoxin activity may take the form of a greater number of cartridges, larger cartridges or other adjunctive therapies. For example, Ferretti et al. pursued a strategy for use of the PMX cartridge wherein daily 2-h PMX cartridge treatments were given until a normalized EAA $(<0.40)$ was achieved. This approach was in a study of 17 post-surgical patients with septic shock. Patients with EAA $>0.6$ were treated until they achieved a normal level of EAA $<0.40$. A group of eight patients with a mean EAA of 0.85 required three hemoperfusion sessions, while a group of two patients with a mean EAA of 0.99 required four hemoperfusion sessions. All patients survived to 28 days [20]. Analysis of other databases containing similar patients to this study population, such as in the existing worldwide registry of patients receiving the PMX cartridge, the EUPHAS2 Registry, found that 80 patients treated with PMX where EAA was measured as EAA $0.6-0.89$ versus 29 patients treated with PMX with EAA > 0.9 showed mortality of $51 \%$ versus 55\%, respectively [21]. Also, Iwagami et al. used a propensity-matched technique to evaluate PMX cartridge use in septic shock patients receiving continuous renal replacement therapy. The 28-day mortality was 40.2\% (393/978) for the PMX group versus $46.8 \%$ (458/978) for the control $(P=0.0003)$. In the patients receiving two PMX cartridge sessions there was a lower 28-day mortality [35.7\% (124/347)] versus those receiving only one session $[42.6 \%(269 / 631)]$ showing a potential "dose response" [22]. A future trial to examine the treatment response effects based on EAA could take the form of a response-adaptive randomization whereby treatments are continued or changed based on clinical and/

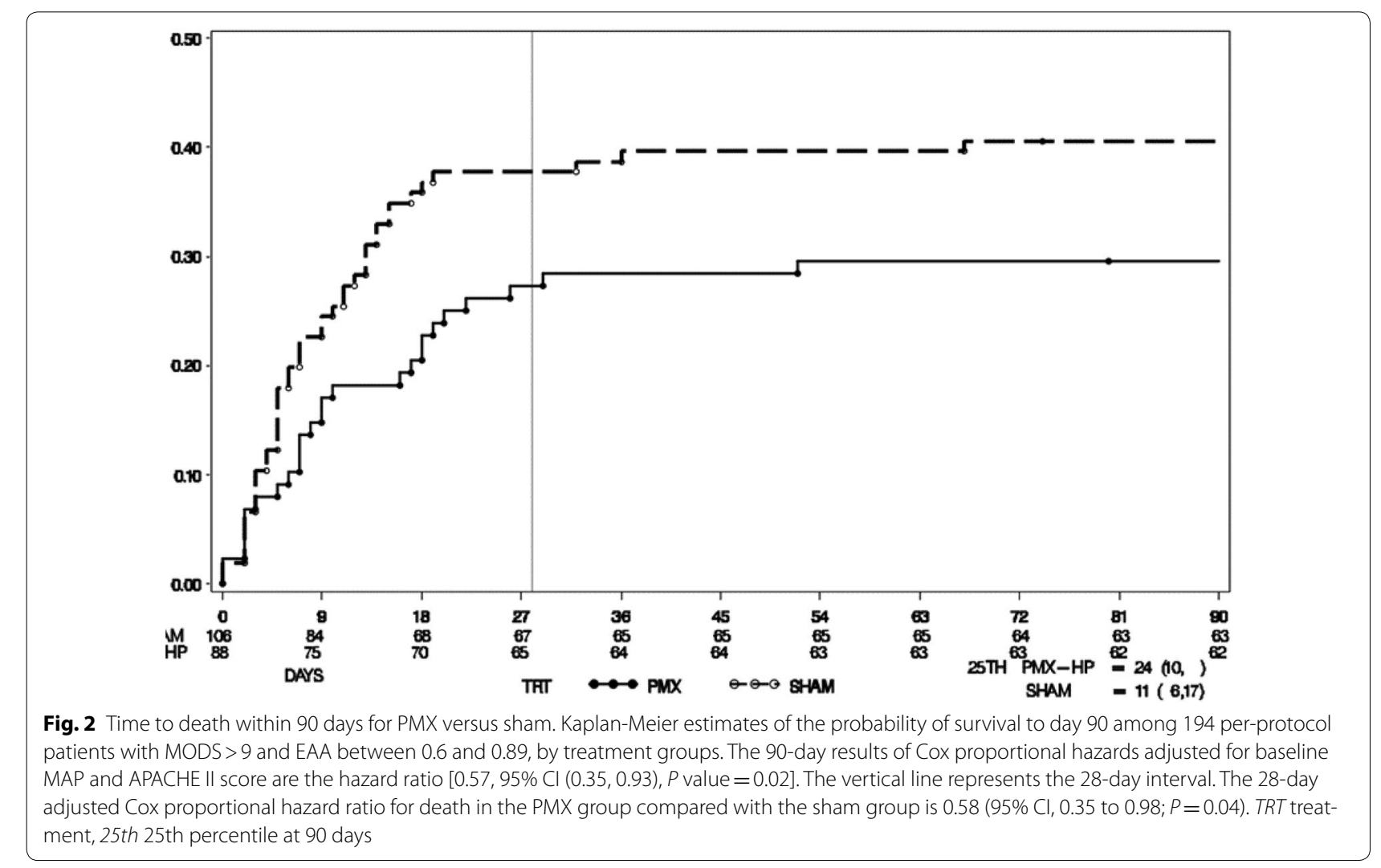


or biomarker changes. In addition, our study findings are consistent with results of recent meta-analyses and large clinical experience in over 100,000 patients treated with PMX to date [23]. In the most recently published systematic review by Chang et al. there was a pooled risk ratio for overall mortality of 0.81 (95\% CI, 0.70-0.95), favoring PMX $(P=0.004)$ across 17 trials [24].

Several other adjunctive therapies have been or continue to be developed to target refractory shock. Corticosteroids have in some studies shown promise albeit with controversy $[25,26]$. In the EUPHRATES study, the majority of patients were receiving corticosteroids based on investigator discretion in a balanced fashion. Other developments include angiotensin II, cytokine removal devices and mechanical support devices. Unique to this therapy is the theragnostic approach of measuring a known mediator of shock and specifically targeting it when it is present at particular levels.

It has been noted that a reduction in EAA regardless of treatment with PMX was associated with a survival benefit and that there was little difference in overall EAA improvement in patients treated with PMX versus sham. Despite this, treatment with PMX in the group with an EAA 0.60-0.89 was associated with a $10.7 \%$ survival benefit. The reasons for this finding are complex and yet to be completely understood. However, we know that the PMX cartridge is able to effectively remove endotoxin from blood in endotoxemic patients $[9,13]$. The biology of endotoxin in humans is characterised by movement through different compartments, which presumably could occur following LPS removal by the PMX cartridge. Srisawat et al. recently demonstrated a similar finding with modulation of HLA-DR that was statistically significant in patients with septic shock treated with PMX versus sham, but again no significant difference in post-treatment EAA [27].

There are important limitations to consider in this post hoc analysis. The analysis of the EUPHRATES study population with baseline EAA 0.60-0.89 must be considered as hypothesis generating only. Second, we have limited information to understand what contributes to extreme endotoxemia $(E A A \geq 0.9)$. Potential options include inadequate source control, development of secondary hospital-acquired infections, baseline susceptibility and genetic profile or profound defects in endogenous endotoxin clearance mechanisms such as liver cirrhosis [9]. The study did not retain plasma or serum for subsequent analysis of cytokines or other molecular components yet these studies will surely be explored in future work.

In conclusion, these hypothesis-generating findings, based on a post hoc exploratory analysis of the EUPHRATES trial, suggest measurable responses in patients with septic shock and an EAA $\geq 0.6$ to 0.89 on changes in mean arterial pressure, ventilator-free days and mortality. Future study is planned to validate this result.

\section{Electronic supplementary material}

The online version of this article (https://doi.org/10.1007/s00134-018-5463-7) contains supplementary material, which is available to authorized users.

\section{Author details \\ ${ }^{1}$ Department of Critical Care, St. Michael's Hospital, University of Toronto, 4-054c Donnelly Wing, Toronto, ON M5B1W8, Canada. ${ }^{2}$ Spectral Medical Inc, Toronto, Canada. ${ }^{3}$ Department of Critical Care Medicine, Faculty of Medicine and Dentistry, University of Alberta, Edmonton, Canada. ${ }^{4}$ AMAREX Clinical Research, Germantown, MD, USA. ${ }^{5}$ Fondazione Policlinico Universitario A. Gemelli IRCCS-Universitá Cattolica del Sacro Cuore, Rome, Italy.}

\section{Acknowledgements}

The authors thank all of the investigators, coordinators, patients and other participants who contributed to the EUPHRATES trial. Dr. Bagshaw is supported by a Canada Research Chair in Critical Care Nephrology.

\section{Funding}

This trial was funded by Spectral Medical Inc.

\section{Compliance with ethical standards}

\section{Conflicts of interest}

Dr. Klein served as the medical monitor for the sponsor for the EUPHRATES trial and is a consultant to Spectral Medical. Dr. Walker and D. Foster are employees of Spectral Medical. H. Mekonnen is an employee of Amarex Clinical Research LLC, the contract research organization that ran the EUPHRATES trial.

\section{Open Access}

This article is distributed under the terms of the Creative Commons Attribution-NonCommercial 4.0 International License (http://creativecommons.org/ licenses/by-nc/4.0/), which permits any noncommercial use, distribution, and reproduction in any medium, provided you give appropriate credit to the original author(s) and the source, provide a link to the Creative Commons license, and indicate if changes were made.

Received: 14 September 2018 Accepted: 12 November 2018 Published online: 23 November 2018

\section{References}

1. Danner RL, Elin RJ, Hosseini JM, Wesley RA, Reilly JM, Parillo JE (1991) Endotoxemia in human septic shock. Chest 99(1):169-175

2. Angus DC, Birmingham MC, Balk RA et al (2000) E5 murine monoclonal antiendotoxin antibody in gram-negative sepsis: a randomized controlled trial. E5 Study Investigators. JAMA 283(13):1723-1730

3. Dellinger RP, Tomayko JF, Angus DC et al (2009) Efficacy and safety of a phospholipid emulsion (GR270773) in Gram-negative severe sepsis: results of a phase II multicenter, randomized, placebo-controlled, dosefinding clinical trial. Crit Care Med 37(11):2929-2938

4. Levin M, Quint PA, Goldstein B et al (2000) Recombinant bactericidal/ permeability-increasing protein ( $\mathrm{BBPI} 21)$ as adjunctive treatment for children with severe meningococcal sepsis: a randomised trial. rBPI21 Meningococcal Sepsis Study Group. Lancet 356(9234):961-967

5. McCloskey RV, Straube RC, Sanders C, Smith SM, Smith CR (1994) Treatment of septic shock with human monoclonal antibody HA-1A. A randomized, double-blind, placebo-controlled trial. CHESS Trial Study Group. Ann Intern Med 121(1):1-5

6. Opal SM, Laterre PF, Francois B et al (2013) Effect of eritoran, an antagonist of MD2-TLR4, on mortality in patients with severe sepsis: the ACCESS randomized trial. JAMA 309(11):1154-1162 
7. Marshall JC, Foster D, Vincent JL et al (2004) Diagnostic and prognostic implications of endotoxemia in critical illness: results of the MEDIC study. J Infect Dis 190(3):527-534

8. Shoji H (2003) Extracorporeal endotoxin removal for the treatment of sepsis: endotoxin adsorption cartridge (Toraymyxin). Ther Apher Dial 7(1):108-114. https://doi.org/10.1046/j.1526-0968.2003.00005.x

9. Ronco C, Klein DJ (2014) Polymyxin B hemoperfusion: a mechanistic perspective. Crit Care 9;18(3):309

10. Cruz DN, Antonelli M, Fumagalli R et al (2009) Early use of polymyxin B hemoperfusion in abdominal septic shock: the EUPHAS randomized controlled trial. JAMA 301(23):2445-2452

11. Payen DM, Guilhot J, Launey Y et al (2015) Early use of polymyxin B hemoperfusion in patients with septic shock due to peritonitis: a multicenter randomized control trial. Intensive Care Med 41(6):975-984

12. Klein DJ, Foster D, Schorr CA, Kazempour K, Walker PM, Dellinger RP (2014) The EUPHRATES trial (Evaluating the use of polymyxin B hemoperfusion in a randomized controlled trial of adults treated for endotoxemia and septic shock): study protocol for a randomized controlled trial. Trials 15:218. https://doi.org/10.1186/1745-6215-15-218

13. Dellinger RP, Bagshaw SM, Antonelli M et al (2018) Targeted Polymyxin-B hemoperfusion for the treatment of endotoxemia and septic shock: a multicenter randomized controlled trial (EUPHRATES). JAMA 20(14):14551463. https://doi.org/10.1001/jama.2018.14618

14. Pocock Stuart J, Stone Gregg W (2016) The primary outcome fails-now what? N Engl J Med 375:861-870. https://doi.org/10.1056/NEJMra1510 064

15. Romaschin AD, Obiezu-Forster CV, Shoji H, Klein DJ (2017) Novel insights into the direct removal of endotoxin by polymyxin B hemoperfusion. Blood Purif 44(3):193-197

16. Dünser MW, Ruokonen E, Pettilä V, Ulmer H, Torgersen C, Schmittinger CA, Jakob S, Takala J (2009) Association of arterial blood pressure and vasopressor load with septic shock mortality: a post hoc analysis of a multicenter trial. Crit Care 13(6):R181

17. Standford Lab_Lab Test Guide. https://www.stanfordlab.com/LabTe stGuide/. Accessed 01 May 2018
18. Moya-Horno I, Viteri S, Karachaliou N, Rosell R (2018) Combination of immunotherapy with targeted therapies in advanced non-small cell lung cancer (NSCLC). Ther Adv Med Oncol. https://doi.org/10.1177/17588 34017745012

19. Reck M, Rabe KF (2017) Precision diagnosis and treatment for advanced non-small-cell lung cancer. N Engl J Med 377:849-861

20. Ferretti G, Ruberto F, Morabito V, Pugliese F (2010) Early management of endotoxemia using the endotoxin activity assay and polymyxin B-based hemoperfusion. Contrib Nephrol 167:91-101

21. Cutuli SL, Artigas A, Fumagalli R et al (2016) Polymyxin-B hemoperfusion in septic patients: analysis of a multicenter registry. Ann Intensive Care 6(1):77

22. Iwagami M, Yasunaga H, Noiri E, Horiguchi H, Fushimi K, Matsubara T, Yahagi N, Doi K (2016) Potential survival benefit of polymyxin B hemoperfusion in spetic shock patients on continuous renal replacement therapy: a propensity-matched analysis. Blood purif 42:9-17

23. Zhou F, Peng Z, Murugan R, Kellum JA (2013) Blood purification and mortality in sepsis: a meta-analysis of randomized trials. Crit Care Med 41(9):2209-2220

24. Chang T, Tu YK, Lee CT, Chao A, Huang CH, Wang MJ, Yeh YC (2017) Effects of polymyxin $B$ hemoperfusion on mortality in patients with severe sepsis and septic shock: a systemic review, meta-analysis update, and disease severity subgroup meta-analysis. Crit Care Med 45(8):e858-e864

25. Venkatesh B, Finfer S, Cohen J et al (2018) Adjunctive glucocorticoid therapy in patients with septic shock. N Engl J Med 378:797-808. https:// doi.org/10.1056/NEJMoa1705835

26. Suffredini AF (2018) A role for hydrocortisone therapy in septic shock? N Engl J Med 378:860-861

27. Srisawat N, Tungsanga S, Lumlertgul N et al (2018) The effect of polymyxin B hemoperfusion on modulation on human leukocyte antigen DR in severe sepsis patients. Crit Care 22:279. https://doi.org/10.1186/s3054 -018-2077-y 